\title{
A note on spectral sublinearity for collections of positive compact operators on Banach lattices
}

https://doi.org/10.1515/spma-2018-0029

Received December 15, 2017; accepted July 7, 2018

\begin{abstract}
In this paper we consider the question when a triangularizable semigroup $\mathcal{S}$ of positive compact ideal-triangularizable operators on an order continuous Banach lattice $X$ is ideal-triangularizable. We prove that triangularizability always implies ideal-triangularizability iff $X$ contains at most one atom. Under this condition we connect ideal-triangularizability of $\mathcal{S}$ with spectral properties of $\mathcal{S}$. Surprisingly, $\mathcal{S}$ is idealtriangularizable iff the spectral radius is subadditive on $\mathcal{S}$ iff the spectral radius is submultiplicative on $\mathcal{S}$. We also consider a pair of positive compact operators $A$ and $T$ with the property that $A$ has a $T$-stable spectrum.
\end{abstract}

Keywords: Banach lattices, Positive operators, Triangularizability, Stable spectrum

MSC: Primary: 46B42, 47A15 Secondary: 47B65

\section{Introduction}

Throughout the paper we assume that all spaces are at least two-dimensional. Suppose $\mathcal{S}$ is a semigroup of $n \times n$ complex matrices or compact operators on a complex Banach space. Then $S$ is triangularizable under "mild" spectral conditions on $\mathcal{S}$. For example, $\mathcal{S}$ is triangularizable iff the spectrum is sublinear on $\mathcal{S}$ (see [10, Theorem 8.4.7]). One cannot replace sublinearity by subadditivity of the spectrum unless the semigroup $\mathcal{S}$ consists of rank-one operators (see [10, Theorem 8.4.8]). In this paper we find spectral conditions on positive compact operators on Banach lattices which guarantee that the operators are simultaneously ideal-triangularizable.

In Section 3 we connect triangularizability with ideal-triangularizability of semigroups of positive compact operators on order continuous Banach lattices. Since the matrix

$$
\left[\begin{array}{ll}
1 & 1 \\
1 & 1
\end{array}\right]
$$

is ideal-irreducible, it is realistic and necessary to assume that each of the operators in $\mathcal{S}$ is idealtriangularizable in its own right. Even then it can happen that a triangularizable semigroup is idealirreducible (see Example 3.1). The main obstruction here is the atomic lattice structure of $\mathbb{R}^{2}$. If an order continuous Banach lattice has at most one atom, then triangularizability implies ideal-triangularizability (Theorem 3.2) for semigroups of positive compact ideal-triangularizable operators. In Theorem 3.4 we provide necessary and sufficient spectral conditions for a semigroup $\mathcal{S}$ of ideal-triangularizable positive com-

\footnotetext{
`Corresponding Author: M. Kandić: Faculty of Mathematics and Physics, University of Ljubljana, Jadranska 19, SI-1000 Ljubljana, Slovenija Institute of Mathematics, Physics and Mechanics, Jadranska 19, SI-1000 Ljubljana, Slovenija,

E-mail: marko.kandic@fmf.uni-lj.si
} 
pact operators on an order continuous Banach lattice to be simultaneously ideal-triangularizable. One of such conditions is sublinearity of the spectrum.

In Section 4 we restrict ourselves to a pair $\{A, T\}$ of positive compact operators. Since Example 3.1 provides an example of an ideal-irreducible pair of ideal-triangularizable positive operators with a sublinear spectrum, we consider a special type of sublinearity of the spectrum, the so-called "stability of the spectrum". Recall that a bounded operator $A$ on a complex Banach space is said to have a $T$-stable spectrum whenever $r(A+\lambda T) \leq r(A)$ for each $\lambda \in \mathbb{C}$. We prove that a pair $\{A, T\}$ is triangularizable if $A$ is $T$-stable, and moreover, we prove that a pair $\{A, T\}$ is ideal-triangularizable if $A$ is ideal-triangularizable (see Theorem 4.2).

\section{Preliminaries}

Let $X$ be a real vector lattice. A vector $x$ is said to be positive if $x \geq 0$. The set $X_{+}$of all positive vectors is called the positive cone of $X$. Given a vector $x \in X$, the supremum of the set $\{x,-x\}$ is denoted by $|x|$. A Banach lattice $X$ is a Banach space which is also a vector lattice such that $0 \leq\|x\| \leq\|y\|$ whenever $0 \leq|x| \leq|y|$ holds in $X$. We write $x_{\alpha} \downarrow 0$ if a net $\left(x_{\alpha}\right)$ decreases towards its infimum 0 . A Banach lattice $X$ is order continuous whenever $x_{\alpha} \downarrow 0$ implies $x_{\alpha} \rightarrow 0$ in norm.

A positive vector $a$ in a normed lattice $X$ is called an atom whenever $0 \leq x \leq a$ implies $x=\lambda a$ for some $\lambda \geq 0$. When $a$ is an atom, the order ideal generated by $a$ is one-dimensional. In this case, each vector $x \in X$ can be decomposed uniquely as $x=\lambda_{a} a+y$ where $\lambda_{a} \in \mathbb{R}$ and $a \perp y=0$. The mapping $\varphi_{a}: x \mapsto \lambda_{a}$ is called the coordinate or biorthogonal functional associated to the atom $a$. It is well-known that $\varphi_{a}$ is a positive bounded linear functional. Since an atom $a$ and its positive multiple generate the same order ideal, to distinguish between atoms which generate different order ideals we assume that atoms are always unit vectors. If we order the vector space $\mathbb{R}^{n}$ coordinate-wise, then $\mathbb{R}^{n}$ becomes a vector lattice whose positive cone is precisely the set of all vectors with nonnegative coordinates. Furthermore, the pair $\left(\mathbb{R}^{n},\|\cdot\|_{2}\right)$ is a Banach lattice and the set of standard basis vectors in $\mathbb{R}^{n}$ is precisely the set of all atoms of $\mathbb{R}^{n}$. It should be noted that whenever a normed lattice $X$ has at most one atom and $\operatorname{dim} X \geq 2$, then $X$ is infinite-dimensional.

All operators and functionals are always assumed to be linear and bounded. An operator on a vector lattice $X$ is positive if it leaves the positive cone $X_{+}$invariant. In particular, an $n \times n$ matrix as an operator on a vector lattice $\mathbb{R}^{n}$ is positive iff it has nonnegative entries. Such matrices are usually called nonnegative. For $\varphi \in X^{*}$ and $x \in X$ we denote by $\varphi \otimes x$ the rank-one operator on $X$ defined as $(\varphi \otimes x)(y):=\varphi(y) x$.

A family $\mathcal{F}$ of operators on a Banach space $X$ is said to be reducible if there exists a nontrivial closed subspace of $X$ that is invariant under every member of $\mathcal{F}$. Otherwise, we say that $\mathcal{F}$ is irreducible. If there exists a maximal chain of closed subspaces whose elements are invariant under every member of $\mathcal{F}$, then $\mathcal{F}$ is said to be triangularizable. A family $\mathcal{F}$ of operators on a Banach lattice is said to be ideal-reducible if there exists a nontrivial closed ideal of $X$ which is invariant under every operator in $\mathcal{F}$. Otherwise, we say that $\mathcal{F}$ is ideal-irreducible. A family $\mathcal{F}$ is ideal-triangularizable if there is a chain $\mathcal{C}$ that is maximal as a chain of closed ideals of $X$ and that has the property that every ideal in $\mathcal{C}$ is invariant under all operators in $\mathcal{F}$. By [4, Proposition 2.1], ideal-triangularizable family of operators is always triangularizable.

Let $X$ be a complex Banach space. The spectrum and the spectral radius of an operator $T$ are denoted by $\sigma(T)$ and $r(T)$, respectively. The spectrum is said to be sublinear on a pair $\{S, T\}$ of operators $S, T: X \rightarrow X$ whenever

$$
\sigma(S+\lambda T) \subseteq \sigma(S)+\lambda \sigma(T)
$$

holds for every scalar $\lambda \in \mathbb{C}$. If the spectrum is sublinear on every pair $\{S, T\}$ from a given family $\mathcal{F}$ of operators on $X$, then we say that the spectrum is sublinear on $\mathcal{F}$. Subadditivity and submultiplicativity of the spectrum are defined analogously. Similarly, one can define sublinearity, subadditivity and submultiplicativity of the spectral radius. Spectral conditions of positive operators on Banach lattices are considered to be spectral conditions of the corresponding extensions to the Banach lattice compactification. 
For not explained terminology on vector and Banach lattices we refer the reader to [2]. For the treatise on ideal-reducibility we refer the reader to [1]. For the results on triangularizability and ideal-triangularizability we refer the reader to [10], [4], [3] and [5].

\section{When does triangularizability implies ideal-triangularizability?}

Suppose $\mathcal{S}$ is a semigroup of compact operators on a Banach space $X$. As it was already mentioned, triangularizability of $\mathcal{S}$ is deeply connected to some special spectral properties of the semigroup $\mathcal{S}$. For example, $\mathcal{S}$ is triangularizable iff the spectrum is sublinear on $\mathcal{S}$ iff commutators of members from $\mathcal{S}$ are quasinilpotent. For details see [10, Theorem 8.4.7 and Theorem 8.5.2].

Assume now that $X$ is a Banach lattice and $S$ is a semigroup of positive compact operators on $X$. The natural problem is to find spectral conditions on $\delta$ under which $\delta$ is ideal-triangularizable. The following example shows that triangularizability, or equivalently, sublinearity of the spectrum, is not enough to guarantee that $\mathcal{S}$ is ideal-reducible.

Example 3.1. Nonnegative matrices

$$
A=\left[\begin{array}{ll}
1 & 1 \\
0 & 0
\end{array}\right] \quad \text { and } \quad B=\left[\begin{array}{cc}
0 & 0 \\
1 & 1
\end{array}\right]
$$

are simultaneously ideal-irreducible since $(A+B) e_{1}=(A+B) e_{2}=e_{1}+e_{2}$. Since the vector $e_{1}-e_{2}$ is an eigenvector of $A$ and $B$, the semigroup $\mathcal{S}:=\{A, B\}$ is triangularizable. Therefore, $\mathcal{S}$ enjoys many important spectral properties, yet it is ideal-irreducible.

The main obstacle is the lattice structure of $X$, namely the presence of more than one atom.

Theorem 3.2. For an order continuous Banach lattice $X$ the following statements are equivalent.

(i) Every triangularizable semigroup of positive compact ideal-triangularizable operators is idealtriangularizable.

(ii) X has at most one atom.

Proof. (i) $\Rightarrow$ (ii) Suppose $X$ has at least two atoms; denote two of them by $e$ and $f$. Let $\varphi_{e}$ and $\varphi_{f}$ be the biorthogonal functionals associated to $e$ and $f$, respectively. Define operators $S:=\left(\varphi_{e}+\varphi_{f}\right) \otimes e$ and $T:=\left(\varphi_{e}+\varphi_{f}\right) \otimes f$. Then $S^{2}=S, T^{2}=T, S T=S$ and $T S=T$, so that $S:=\{S, T\}$ is a semigroup of positive compact operators on $X$. It is easy to see that the subspace $\mathcal{M}_{1}$ spanned by the vector $e-f$ is invariant under $\mathcal{S}$. Obviously, the subspace $\mathcal{M}_{2}$ spanned by $e$ and $f$ is also invariant under $\mathcal{S}$. Denote by $\pi$ the quotient projection from $X$ onto $X / \mathcal{M}_{2}$. Since $X / \mathcal{M}_{2}$ is a Banach space and the semigroup $\mathcal{S}$ induces the zero semigroup on $X / \mathcal{M}_{2}$, any maximal chain $\mathcal{C}$ of closed subspaces of $X / \mathcal{M}_{2}$ rises to the triangularizing chain $\mathcal{C}_{0}:=\left\{\{0\}, \mathcal{M}_{1}\right\} \cup\left\{\pi^{-1}(\mathcal{M}): \mathcal{M} \in \mathcal{C}\right\}$ for the semigroup $\mathcal{S}$. By the assumption, $\mathcal{S}$ is ideal-triangularizable. By [7, Proposition 2.3] the restriction of $\mathcal{S}$ to $\mathcal{M}_{2}$ is also ideal-triangularizable. Since $\mathcal{M}_{2}$ is atomic, one of atoms $e$ and $f$ should be an eigenvector for both $S$ and $T$. This is clearly not the case. Hence, if (i) holds, $X$ has at most one atom.

(ii) $\Rightarrow$ (i) Let $\mathcal{S}$ be a triangularizable semigroup of positive compact ideal-triangularizable operators on $X$ which has at most one atom. If $\mathcal{J}$ is a closed ideal in $X$, then $\mathcal{J}$ and $X / \mathcal{J}$ have at most one atom. Hence, by the Ideal-Triangularization lemma it suffices to prove that $\mathcal{S}$ is ideal-reducible.

Clearly we may assume $\mathcal{S} \neq\{0\}$. If $X$ has no atoms, then every operator in $\mathcal{S}$ is quasinilpotent by [5, Proposition 4.5], so that $S$ is ideal-triangularizable by [3, Theorem 4.5].

Assume that $X$ contains precisely one atom $a$. Since $\mathcal{S}$ is triangularizable, the spectral radius is submultiplicative on $\mathcal{S}$. If there exists a quasinilpotent operator $S$ in $\mathcal{S}$, then the semigroup ideal generated by $S$ is ideal-reducible by [3, Theorem 4.5], which implies that $S$ is ideal-reducible by [5, Proposition 2.1]. 
Assume now that every nonzero operator in $\mathcal{S}$ is non-quasinilpotent. If there exist nonzero operators $A, B \in \mathcal{S}$ with $A B=0$, then the equality

$$
(B S A)^{2}=(B S) A B(S A)=\{0\}
$$

implies $B S A=\{0\}$. Ideal-reducibility of $\mathcal{S}$ follows again from [5, Proposition 2.1].

Assume now that $\mathcal{S}$ has no nontrivial zero-divisors. Then the set $\mathcal{T}=\mathcal{S} \backslash\{0\}$ is a semigroup of positive ideal-triangularizable operators with the property that

$$
a \notin Z(S):=\left\{e \text { is an atom in } X: \varphi_{e}(S e)=0\right\}
$$

for all $S \in \mathcal{T}$. Indeed, by [8, Proposition 4], we have $\sigma(S) \backslash\{0\}=\left\{\varphi_{a}(S a)\right\} \backslash\{0\}$. Since $S$ is not quasinilpotent, we have $\varphi_{a}(S a)=r(S) \neq 0$. In this case, ideal-reducibility of $\mathcal{S}$ follows from [5, Proposition 4.4].

The following theorem will be needed in the proof of Theorem 3.4. We omit its proof since it follows the same lines as the proof of [10, Theorem 8.7.15].

Theorem 3.3. If a spectral radius is submultiplicative but not multiplicative on a semigroup $\mathcal{S}$ of positive compact operators on a Banach lattice, then the semigroup is ideal-reducible.

If $X$ is a Banach space and $S$ is a triangularizable semigroup of compact operators on $X$, then the spectrum is always subadditive on $\mathcal{S}$. However, [10, Example 4.2.6] shows that the converse implication does not hold. On the other hand, semigroups of operators of rank at most one with subadditive spectrum are triangularizable by [10, Theorem 4.2.5]. For ideal-triangularizable positive compact operators on order continuous Banach lattices with at most one atom the situation improves: subadditivity of the spectral radius implies idealtriangularizability of the whole semigroup.

Theorem 3.4. Let $\mathcal{S}$ be a semigroup of positive compact ideal-triangularizable operators on an order continuous Banach lattice $X$ with at most one atom. The following assertions are equivalent:

(i) $S$ is ideal-triangularizable.

(ii) $S$ is triangularizable.

(iii) Spectrum is sublinear on $\mathcal{S}$.

(iv) Spectrum is subadditive on $\mathcal{S}$.

(v) Spectrum is submultiplicative on $\delta$.

(vi) Spectral radius is subadditive on $\mathrm{S}$.

(vii) Spectral radius is submultiplicative on $\mathcal{S}$.

(viii)Spectral radius is permutable on $\mathrm{S}$.

Proof. By Theorem 3.2, (i) and (ii) are equivalent, and that (ii) implies all statements (iii)-(viii) follows from the Spectral mapping theorem for families of compact operators (see [10, Theorem 7.2.6]).

If $X$ contains no atoms, every operator in $\mathcal{S}$ is quasinilpotent, so that $\mathcal{S}$ is ideal-triangularizable. Since $\mathcal{S}$ consists of quasinilpotent operators, all statements (i)-(viii) trivially hold. Hence, we may assume that $X$ contains precisely one atom $a$.

By [10, Theorem 8.4.3], (iii) implies (ii), and the equivalence between (vii) and (viii) follows from [10, Theorem 8.6.3]. Implications (iii) $\Rightarrow$ (iv), (iv) $\Rightarrow$ (vi) and (v) $\Rightarrow$ (vii) are obvious.

(vi) $\Rightarrow$ (vii) Suppose that the spectral radius is subadditive on $\delta$. By an easy induction one can prove that for each positive operator $S$ on $X$ we have $S^{n} a \geq \varphi_{a}(S a)^{n} a$ from where we conclude $r(S) \geq \varphi_{a}(S a)$. Pick $S$ and $T \in \mathcal{S}$. Since each operator in $\mathcal{S}$ is ideal-triangularizable, we have $r(T)=\varphi_{a}(T a), r(S)=\varphi_{a}(S a)$ and $\varphi_{a}(S T a)=r(S T)=r(T S)=\varphi_{a}(T S a)$. Since

$$
\begin{aligned}
(r(S) & +r(T))^{2} \geq(r(S+T))^{2}=r\left((S+T)^{2}\right) \geq \varphi_{a}\left((S+T)^{2} a\right) \\
& \geq\left(\varphi_{a}((S+T) a)\right)^{2}=\varphi_{a}(S a)^{2}+\varphi_{a}(S T a)+\varphi_{a}(T S a)+\varphi(T a)^{2} \\
& =r(S)^{2}+2 r(S T)+r(T)^{2},
\end{aligned}
$$


we conclude $r(S T) \leq r(S) r(T)$.

(vii) $\Rightarrow$ (i) We prove first that submultiplicativity of the spectral radius implies ideal-reducibility of the semigroup.

If $X$ contains no atoms, then every operator in $\mathcal{S}$ is quasinilpotent and hence $\mathcal{S}$ is ideal-triangularizable. Assume $X$ has a precisely one atom $a$. Then a similar argument as in the proof of Theorem 3.2 yields that for each operator $S \in \mathcal{S}$ we have $r(S)=\varphi_{a}(S a)$. Since the spectral radius is submultiplicative on $\mathcal{S}$, we conclude that the mapping $\phi_{a, a}: S \mapsto \varphi_{a}(S a)$ is submultiplicative on $\mathcal{S}$. Since $\phi_{a, a}$ is always supermultiplicative (see e.g. [8]), the mapping $\phi_{a, a}$ is multiplicative on $\mathcal{S}$, and so ideal-reducibility of $\mathcal{S}$ follows from [8, Theorem 1].

Let $\mathcal{J} \subseteq \mathcal{J}$ be two closed ideals invariant under $\mathcal{S}$. Every operator in the semigroup $\widehat{\mathcal{S}}$ induced by $\mathcal{S}$ on $\mathcal{J} / \mathcal{J}$ is a positive compact operator that is also ideal-triangularizable by [7, Proposition 2.3].

If $X$ contains at most one atom, then $\mathcal{J}$ contains at most one atom as does $\mathcal{J} / \mathcal{J}$. If we prove that the spectral radius is submultiplicative on the semigroup $\widehat{\mathcal{S}}$ induced by $\mathcal{S}$ on $\mathcal{J} / \mathcal{J}$, then the Ideal-Triangularization lemma is applicable, and the proof will be complete.

If $\mathcal{J} / \mathcal{J}$ contains no atoms, then every operator in $\widehat{\mathcal{S}}$ is quasinilpotent, so that the spectral radius is trivially submultiplicative on $\widehat{\mathcal{S}}$.

Suppose that $\mathcal{J} / \mathcal{J}$ contains precisely one atom $a$. The chain $\{0\} \subseteq \mathcal{J} \subseteq \mathcal{J} \subseteq X$ is a complete chain of closed ideals invariant under the semigroup $\mathcal{S}$. Pick $S \in \mathcal{S}$ and denote by $S_{\mathcal{J}}$ and $S_{X}$ operators on $\mathcal{J}$ and $X / \mathcal{J}$ induced by $S$, respectively. Ringrose's theorem for complete chains ([10, Theorem 7.2.7]) implies

$$
\sigma(S) \cup\{0\}=\{0\} \cup \sigma\left(S_{\mathcal{J}}\right) \cup \sigma(\widehat{S}) \cup \sigma\left(S_{X}\right) .
$$

Since $X / \mathcal{J}$ and $\mathcal{J}$ contain no atoms, we have $r(S)=r(\widehat{S})$ for every operator $S \in \mathcal{S}$. This proves that spectral radius is submultiplicative on $\widehat{S}$.

A thorough inspection of the proof of Theorem 3.4 shows that we may add the following equivalent assertions to the list in Theorem 3.4:

(v') Spectrum is multiplicative on $\mathcal{S}$.

(vi')Spectral radius is additive on $\mathcal{S}$.

(vii')Spectral radius is multiplicative on $\mathcal{S}$.

Corollary 3.5. Let $\mathcal{S}$ be a semigroup of positive ideal-triangularizable operators on an order continuous Banach lattice $X$ with at most one atom. Suppose that $\mathcal{S}$ contains a nonzero compact operator. Then $\mathcal{S}$ is ideal-reducible in any of the following cases.

(i) $\mathcal{S}$ is triangularizable.

(ii) Spectral radius is subadditive on $\mathcal{S}$.

(iii) Spectral radius is submultiplicative on $\delta$.

(iv) Spectral radius is permutable on $\mathcal{S}$.

Proof. If $S \in \mathcal{S}$ is a nonzero compact operator, then in each of the cases (i)-(iv) the semigroup ideal generated by $S$ in $\mathcal{S}$ is ideal-triangularizable by Theorem 3.4. Ideal-reducibility of $\mathcal{S}$ now follows from [5, Proposition 2.1].

Let $(\Omega, \mathcal{A}, \mu)$ be a measurable space with a positive measure. It is well-known that when $1 \leq p<\infty$ the Banach lattice $L_{p}(\mu)$ is order continuous. Therefore, Theorem 3.4 and Corollary 3.5 are, in particular, applicable to $L_{p}$ spaces when $1 \leq p<\infty$ and when $\mathcal{A}$ contains at most one atom of the measure $\mu$.

Unfortunately, Theorem 3.4 and Corollary 3.5 are not applicable in the case of nonnegative $n \times n$ matrices since the vector lattice $\mathbb{R}^{n}$ has precisely $n$ atoms.

\section{Operators with stable spectrum}

As we have seen in Example 3.1, a triangularizable semigroup $\{A, T\}$ of ideal-triangularizable positive compact operators on a Banach lattice can be ideal-irreducible. Example 3.1 is not in contradiction with Theo- 
rem 3.4 since the underlying Banach lattice has two atoms. In this short section we prove that a pair $\{A, T\}$ of positive compact ideal-triangularizable operators on a Banach lattice is simultaneously ideal-triangularizable whenever $A$ is $T$-stable (see Theorem 4.2).

Before we proceed to results of this section, we would like to explain the connection between stability and sublinearity of the spectrum. If $A$ has a $T$-stable spectrum, then $T$ is necessarily quasinilpotent by [9, Lemma 3.6]. Moreover, if $\sigma(A)$ has no interior points, then [9, Lemma 3.9] implies $\sigma(A+\lambda T)=\sigma(A)$ for all $\lambda \in \mathbb{C}$, so that the pair $\{A, T\}$ has a sublinear spectrum. On the other hand, if $T$ is quasinilpotent and the pair $\{A, T\}$ has a sublinear spectrum, then it is not hard to see that $A$ is $T$-stable.

We continue with the following lemma that is of independent interest.

Lemma 4.1. Let $A$ and $T$ be positive operators on a Banach lattice $X$ with $T \neq 0$. Suppose $A$ is power compact and has a T-stable spectrum. Then the following statements hold:

(i) $A$ is ideal-reducible.

(ii) If $T$ is compact, then the pair $\{A, T\}$ is ideal-reducible.

Proof. Since $0 \leq A, T \leq A+T$, the pair $\{A, T\}$ is ideal-reducible iff the operator $A+T$ is ideal-reducible.

(i) Suppose $A$ is ideal-irreducible. Since $0 \leq A \leq A+T$ and $r(A+T)=r(A)$, by [6, Corollary 3.5] we conclude $A+T=A$ and so $T=0$, which is in contradiction with the assumption $T \neq 0$.

(ii) If $A$ is a power compact operator, then compactness of $T$ yields that the operator $A+T$ is power compact as well. If $A+T$ would be ideal-irreducible, then the same proof as in (i) would yield $T=0$.

Assume that $A$ and $T$ are positive compact operators on a Banach lattice $X$ such that $A$ has a $T$-stable spectrum. Then the pair $\{A, T\}$ is reducible. Indeed, if $T \neq 0$, then Lemma 4.1(ii) is applicable. Otherwise, $T=0$ and every closed subspace invariant under $A$ is clearly invariant under the zero operator $T$. Similar argument shows that the pair $\{A, T\}$ is ideal-reducible whenever $A$ is ideal-reducible.

Theorem 4.2. Let $A$ and $T$ be positive compact operators on a Banach lattice X. Suppose A has a T-stable spectrum. Then the following statements hold:

(i) The pair $\{A, T\}$ is triangularizable.

(ii) If $A$ is ideal-triangularizable, then the pair $\{A, T\}$ is ideal-triangularizable.

Proof. The remark preceding the theorem implies that the pair $\{A, T\}$ is always reducible, and ideal-reducible whenever $A$ is ideal-reducible. Then (i) and (ii) will follow by invoking the Triangularization and the IdealTriangularization lemma, respectively, once we prove that the property of having a $T$-stable spectrum is inherited by quotients.

Assume $\mathcal{J} \subseteq \mathcal{J}$ are closed subspaces invariant under compact operators $C$ and $D$ on a Banach space $Y$. Assume that $C$ is $D$-stable. By $\widehat{C}$ and $\widehat{D}$ we denote operators induced by $C$ and $D$ on the quotient Banach space $\mathcal{J} / \mathcal{J}$, respectively. Since $\sigma(C)$ has no interior points, for all $\lambda \in \mathbb{C}$ we have $\sigma(C+\lambda D)=\sigma(C)$ by [9, Lemma 3.9]. Since $D$ is quasinilpotent, the spectrum is sublinear on the pair $\{C, D\}$. By [10, Corollary 8.4.2], the spectrum is also sublinear on the pair $\{\widehat{C}, \widehat{D}\}$. Since $\widehat{D}$ is quasinilpotent, it follows that $\sigma(\widehat{C}+\lambda \widehat{D}) \subseteq \sigma(\widehat{C})$ for all $\lambda \in \mathbb{C}$. This yields $r(\widehat{C}+\lambda \widehat{D}) \leq r(\widehat{C})$ for every $\lambda \in \mathbb{C}$ and the proof is finished.

We conclude this paper with an example of an irreducible pair $\{A, T\}$ of ideal-triangularizable $3 \times 3$ matrices such that $A$ is $T$-stable, $T$ is $A$-stable and only one of them is nonnegative.

Example 4.3. Consider the vector space $\mathcal{S}$ of $3 \times 3$ complex matrices spanned by

$$
A=\left[\begin{array}{ccc}
0 & 1 & 0 \\
0 & 0 & -1 \\
0 & 0 & 0
\end{array}\right] \quad \text { and } \quad T=\left[\begin{array}{ccc}
0 & 0 & 0 \\
1 & 0 & 0 \\
0 & 1 & 0
\end{array}\right]
$$

As it is shown in [10, Example 1.7.11], $\mathcal{S}$ consists of nilpotent matrices and $\mathcal{S}$ does not have a nontrivial invariant subspace. Since $\mathcal{S}$ consists of nilpotent matrices, $A$ is $T$-stable and $T$ is $A$-stable. 
Acknowledgement: The author would like to express his gratitude to both anonymous referees whose comments and suggestions significantly improved the exposition of the paper.

The author acknowledges financial support from the Slovenian Research Agency, Grants No. P1-0222 and J1-8133.

\section{References}

[1] Y. Abramovich and C.D. Aliprantis, An invitation to operator theory, Vol. 50. Providence, RI: American Mathematical Society, 2002.

[2] C.D. Aliprantis and O. Burkinshaw, Positive operators, 2nd edition, Springer 2006.

[3] R. Drnovšek, Common invariant subspaces for collections of operators, Integral Equat. Oper. Th. 39 (2001), 253-266.

[4] R. Drnovšek, Triangularizing semigroups of positive operators on an atomic normed Riesz spaces, Proc. Edin. Math. Soc. 43 (2000), 43-55.

[5] R. Drnovšek, M. Kandić, Ideal-triangularizability of semigroups of positive operators, Integral Equat. Oper. Th. 64 (2009), no. 4, 539-552.

[6] N. Gao, Extensions of Perron-Frobenius theory, Positivity 17 (2013), 965-977.

[7] M. Kandić, Ideal-triangularizability of upward directed sets of positive operators, Ann. Funct. Anal. 2 (2011), no. 1, $206-219$.

[8] M. Kandić, Multiplicative coordinate functionals and ideal-triangularizability, Positivity 17 (2013), 1085-1099.

[9] M. Kennedy and H. Radjavi, Spectral conditions on Lie and Jordan algebras of compact operators, J. Funct. Anal. 256 (2009), 3143-3157.

[10] H. Radjavi and P. Rosenthal, Simultaneous Triangularization, Springer-Verlag, New York, 2000. 